\title{
Habitability in brown dwarf systems
}

Emeline Bolmont

\begin{abstract}
The very recent discovery of planets orbiting very low mass stars sheds light on these exotic objects. Planetary systems around low-mass stars and brown dwarfs are very different from our solar system: the planets are expected to be much closer than Mercury, in a layout that could resemble the system of Jupiter and its moons. The recent discoveries point in that direction with, for example, the system of Kepler-42 and especially the system of TRAPPIST- 1 which has seven planets in a configuration very close to the moons of Jupiter. Low-mass stars and brown dwarfs are thought to be very common in our neighborhood and are thought to host many planetary systems. The planets orbiting in the habitable zone of brown dwarfs (and very low-mass stars) represent one of the next challenges of the following decades: they are the only planets of the habitable zone whose atmosphere we will be able to probe (e.g. with the JWST).
\end{abstract}

\section{Introduction}

In the $10 \mathrm{pc}$ around us, $75 \%$ of objects are low mass stars and brown dwarfs (RECONS project http: //www.recons.org/, e.g. Henry et al. 2016). It is thought that a majority of those low-mass stars and brown dwarfs (BDs) host planetary systems (e.g. Dressing and Charbonneau 2015). For instance, the population of Earth-size planets in the habitable zone (HZ) of low-mass stars has been estimated to be between $\sim 20 \%$ (Dressing and Charbonneau 2015 ) and $\sim 40-50 \%$ (Bonfils et al. 2013, Dressing and Charbonneau 2013; Kopparapu 2013). The HZ is here defined as the region around a star in which a planet with a sufficiently dense atmosphere can host surface liquid water (e.g. Kasting et al.|1993; Selsis et al.|2007).

Because of the low luminosity of these objects, planets inside the HZ are sufficiently close-in to be influenced by the tidal interactions between the dwarf and the

Laboratoire AIM Paris-Saclay, CEA/Irfu Université Paris-Diderot CNRS/INSU, 91191 Gif-surYvette, France, e-mail: emeline.bolmontecea.fr 
planets (Barnes et al. 2008, 2009, 2010, 2011). Moreover, the spectral distribution of these dwarfs and the proximity of the $\mathrm{HZ}$ would likely cause the climate to be very different from that of the Earth (e.g., Segura et al. 2005; Rauer et al. 2011).

This situation is even more extreme for BDs. They are not massive enough to start the hydrogen fusion reaction (Chabrier and Baraffe 1997, 2000) so their temperature is even cooler than for M-dwarfs and they also cool down with time. Their HZ therefore moves inward and can even be within the Roche limit at late ages. Planets in the $\mathrm{HZ}$ of brown dwarfs should thus be submitted to strong tides (Bolmont et al. 2011) which influence their orbit and rotation.

The existence and fate of planets around BDs has been considered punctually over the years (e.g., the works of Desidera 1999, Andreeshchev and Scalo 2004, Bolmont et al.2011, Barnes and Heller 2013, but the recent discoveries of planetary systems around very low-mass stars (Kepler- 42 , a $0.13 M_{\odot}$ dwarf with at least three small planets: Muirhead et al. 2012; Proxima, a $0.123 M_{\odot}$ dwarf hosting at least a planet: Anglada-Escudé et al. 2016, and TRAPPIST-1, a $\sim 0.08 M_{\odot}$ dwarf with a multiple planet system: Gillon et al. 2016, 2017) have contributed to renew the interest on these objects.

The discovery of the TRAPPIST-1 planets also illustrates the importance to study those objects: indeed those planets are the only known planets of the $\mathrm{HZ}$ for which we will be able to probe the atmosphere with future instruments such as the JWST (e.g. Belu et al. 2013) or the E-ELT (Rodler and López-Morales 2014). Note that other planets around low-mass stars could be targets for the JWST, like LHS 1140b (Dittmann et al. 2017). However due to its high surface gravity and the scarcity of its transits, it would be a much more technically challenging observation than that of the TRAPPIST-1 planets. Note that TRAPPIST- 1 is particularly interesting in the framework of this chapter, because its estimated mass is just above the theoretical limit between BDs and low-mass stars.

We first introduce brown dwarfs and discuss why they are so special in terms of hosting environments for planets and then explore different aspects of the habitability of planets orbiting brown dwarfs.

\section{Brown dwarfs and their evolution}

Before ever actually observing BDs, the existence of these objects has been conceptualized by Kumar (1963) and Hayashi and Nakano (1963) as being failed stars: not massive enough for the core temperature to reach values allowing the fusion of hydrogen. We first give the definition of a BD and then discuss what makes them so special when considering the habitability of potential planets. 


\section{What are brown dwarfs?}

BDs are objects which are thought to form like stars (Luhman 2012), by the gravitational collapse of a molecular cloud (thermal radio jets, typical of young stellar systems, have been detected in young BDs systems. This shows the continuity of the formation processes between low mass stars and BDs; e.g. Morata et al. 2015). However, contrary to stars, these objects are not massive enough for their core temperature to reach the level needed to initiate the hydrogen fusion nuclear reaction (see Luhman 2012 for a review on BDs). They are therefore very faint and were first observed quite recently by Nakajima et al. (1995) and Rebolo et al. (1995).

As the fusion temperature of hydrogen is of about $3 \times 10^{6} \mathrm{~K}$, only objects of more than $M_{\star} \approx 75 M_{\mathrm{J}}$ can initiate the PPI fusion reaction chain. This gives an upper mass for BDs (Chabrier and Baraffe 1997, 2000). The lower limit is however much less well defined. There are some arguments both observational (Caballero et al. 2007) and analytical (Padoan and Nordlund 2004, Hennebelle and Chabrier 2008) which suggest that the same star formation process can produce objects down to a few mass of Jupiter. One possible lower limit definition would be the deuterium fusion limit: studies show that objects of mass higher than $\approx 13 M_{\mathrm{J}}$ can still initiate the deuterium fusion reaction while objects less massive cannot. BDs would therefore be objects in the mass range of 13 to $75 M_{\mathrm{J}}$ and all objects with a mass lower than $13 M_{\mathrm{J}}$ would be planets (IAU definition, see Boss et al. 2007). However this definition is more of an indication rather than a strong astrophysical limit. Indeed, Spiegel et al. (2011) showed that the limit of $13 M_{\mathrm{J}}$ can change between 11 and $16 M_{\mathrm{J}}$ when considering different metallicities for example.

The population of mini brown dwarfs and giant planets (formed in a protoplanetary disk) can have a common mass range. It is therefore fundamental to try to differentiate those two types of astrophysical objects (Leconte et al. 2009, Spiegel et al. 2011; Leconte et al. 2011a).

Since the discovery of the first BDs in 1995, many more have been detected in star forming regions (in the Chamaeleon I cloud: Comerón et al. (2000) and López Martí et al. (2004)), in open clusters (e.g., in the Pleiades: Zapatero Osorio et al. (1997), and in the young open cluster IC 2391: Barrado y Navascués et al. (2001)) and also among field objects (Kirkpatrick et al. 1999; Phan-Bao et al. 2001).

The number of detected BDs have thus risen thanks to observation missions such as 2MASS (Two Micron All Sky Survey, Skrutskie et al.2006) or WISE (Wide-field Infrared Survey Explorer, Cushing et al. 2011). Due to their low temperature, BDs emit principally in the infrared and are therefore detected by instruments probing these wavelengths. As of 2016, the number of BDs (objets of spectral type L, T and $\mathrm{Y}$ ) is of about 2800 (http://www. johnstonsarchive.net/astro/ browndwarflist.html).

Studies based on the Initial Mass Function (e.g. Salpeter 1955) tend to show that the number of low mass objects like BDs should be much higher than more massive stars. Chabrier (2002) revisited these studies focusing on BDs and showed that there should be as many BDs as there are stars. However, thanks to the intensive 
observational efforts, it has been shown that brown dwarfs are more scarce than previously thought. For example, the WISE survey (Kirkpatrick|2013) showed that within 8 parsec, there are 33 BDs and 211 stars (white dwarfs, O, B, A, F, G, K, $\mathrm{M}$ stars), which yield that there is $1 \mathrm{BD}$ for every 6.4 stars (interestingly, there are 4.1 BDs for every G-star). More recently, the RECONS team (Henry et al. 2016) confirmed this tendency showing that there is one BD for every $\sim 10$ stars within 10 parsec.

\section{What is special about them?}

Because BDs cannot initiate the hydrogen fusion reaction in their core, the energy due to this reaction is here missing to prevent the contraction and the cooling down of these objets. However, by definition, BD are massive enough to initiate the deuterium fusion. As this additional source of energy is able to compensate the radiative losses for a while, the contraction is slowed down and radius and luminosity reach a plateau. As the primordial abundance of deuterium is small and the reaction constants are big, this phase lasts only a few million years for massive BDs and about 100 million years when they are close to the deuterium fusion limit. Fig. 1 shows the evolution of the luminosity of several low-mass objects: from planets of $0.01 M_{\odot}$ $\left(\sim 10 M_{\mathrm{J}}\right)$ to low-mass stars of $0.08 M_{\odot}$ passing by BDs.

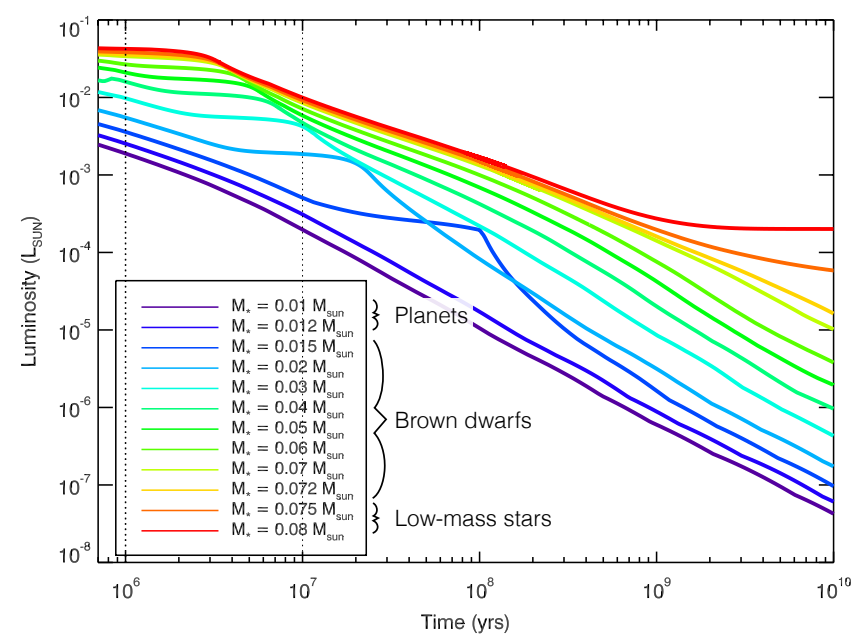

Fig. 1 Evolution of the luminosity of low-mass objects of different masses (grids coming from Leconte et al. 2011b). The two lower mass objects are planets in this model (the limit of deuterium fusion is never reached). The two upper mass objects are low-mass stars (the deuterium is quickly burnt in a few Myr, and the hydrogen fusion begins after a few Gyr). Figure adapted from Bolmont (2013). 


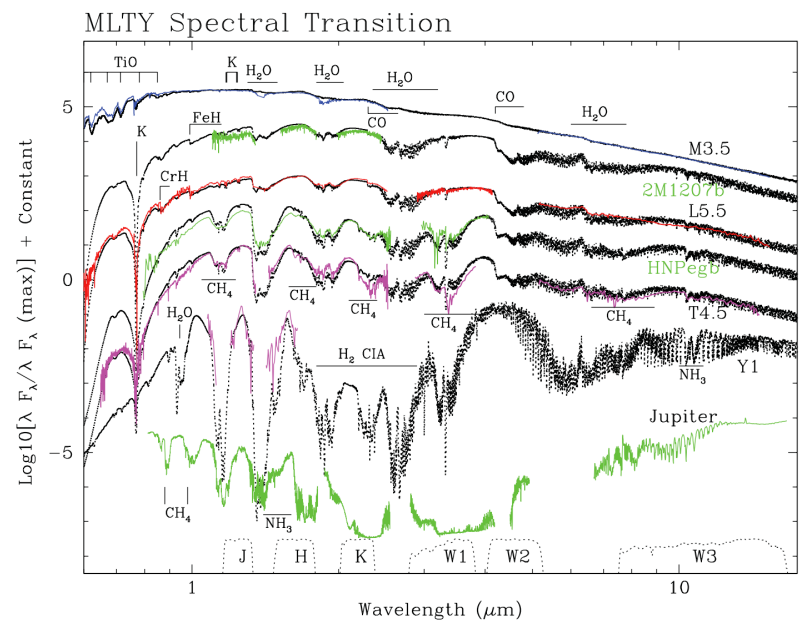

Fig. 2 Spectral Energy Distribution of dwarfs of different spectral types. The full lines represent measurements (using for instance the United Kingdom Infrared Telescope and Spitzer) and the dotted lines are BT-Settl models. For the L, T and Y dwarfs, the $\mathrm{H}_{2} \mathrm{O}$ and $\mathrm{CH}_{4}$ features are well marked. Figure from Allard (2014).

BDs are very cold objects (for example a $5 \mathrm{Gyr}$ old BD of $0.02 M_{\odot}$ has an effective temperature of $\sim 500 \mathrm{~K}$, see the grids of Leconte et al. (2011b) which means that their $\mathrm{HZ}$ is located very close in. The cooling down of these objects also means that the HZ moves in with time, which has a strong importance for the potential habitability of planets.

Finally, their spectra are different from our Sun (Burrows et al. 2000; Allard 2014). Due to their low temperature, their spectra are redshifted and display much more molecular lines than the Sun. Fig. 2 2 shows the spectra of different dwarfs (Spectral types M, L, T and Y). The emission spectrum peaks around 1-2 $\mu \mathrm{m}$ for brown dwarfs, around $0.5 \mu \mathrm{m}$ for the Sun and around $10 \mu \mathrm{m}$ for the Earth. Decreasing the temperature and going from spectral type $\mathrm{M}$ to $\mathrm{Y}$, we can see that the water and methane features become much more visible. These spectral features have impacts on the potential climates of planets. For instance, the ice-albedo feedback, which is a positive feedback (lower temperature $\rightarrow$ more ice $\rightarrow$ higher albedo $\rightarrow$ less absorbed radiation $\rightarrow$ lower temperature) does not occur around red dwarfs due to the much lower value of the albedo of the ice in the IR (Joshi and Haberle 2012).

\section{Habitability of planets around brown dwarfs}

The inward migration of the $\mathrm{HZ}$ as the $\mathrm{BD}$ cools down has a major impact on potentially habitable planets: they might lose water early in their history. Moreover, as BDs are cool objects, the $\mathrm{HZ}$ is close-in and planets are subjected to tides. 


\section{Before reaching the habitable zone}

Planets in the HZ of Gyr-old BDs were initially too hot to host surface liquid water. Fig. 3 shows the evolution of the $\mathrm{HZ}$ for a $0.04 M_{\odot}$ BD. The $\mathrm{HZ}$ moves inward with time so that planets spending some time in the $\mathrm{HZ}$ were all initially too hot for surface liquid water. The closer-in planets spend a lot of time interior to the $\mathrm{HZ}$ (almost 100 Myr for the closest surviving planet in Fig. 3). The farther the planet, the less time it spends interior to the HZ (10 Myr for the farthest planet in Fig. 3 . During this time, all the water is in gaseous form in the atmosphere and is submitted to the high energy radiations from the BD. These radiations can break the water molecules and heat up the upper layers of the atmosphere to drive the escape of the hydrogen and oxygen atoms, which in the end results a net water loss.

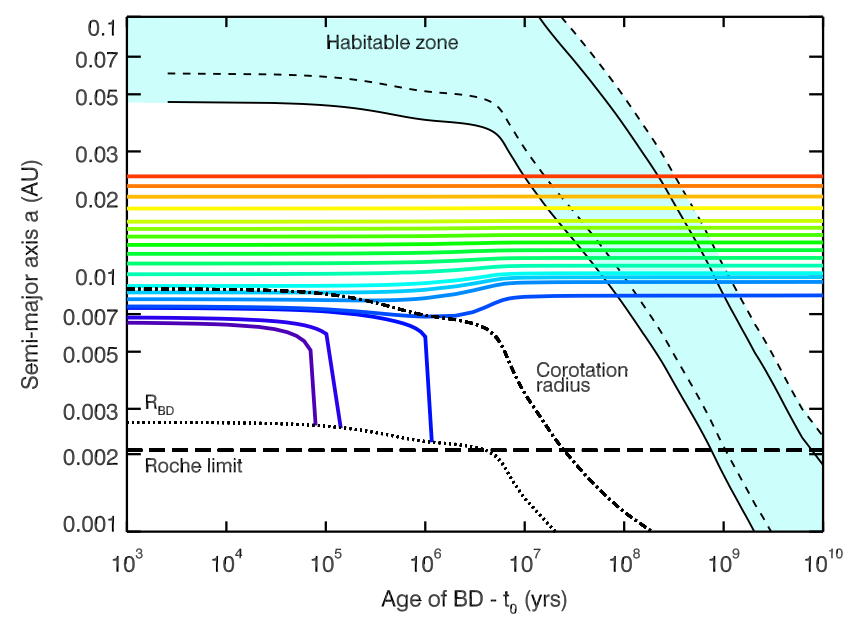

Fig. 3 Tidal evolution of Earth mass planets orbiting a $0.04 M_{\odot}$ BD. The colored lines represent the orbital distance of different planets. The blue shaded area represents the HZ. The dashed-dotted line corresponds to the corotation radius. A planet farther than this limit migrates outward due to the tide it raises in the BD (just like the moon is migrating away from the Earth). The dotted line represents the radius of the BD. The long dashes represent the Roche limit: a planet closer than this limit would be tidally disrupted and create a ring of material around the BD (just like Saturn's rings, which are located inside its Roche limit). Figure adapted from Bolmont et al. (2011).

The mechanisms driving the escape are complex and not well parametrized yet. However, a few studies (Barnes and Heller 2013; Luger and Barnes 2015, Ribas et al. 2016; Bolmont et al. 2017) have tried to give an insight on these phenomenons to estimate the water loss from planets orbiting BDs and low-mass stars. These studies differ on a few hypothesis but all use the variations of the same method which is based on the concept of the energy-limited escape mechanism (Watson et al. 1981; Lammer et al. 2003). This mechanism relies on four steps: 1) the water molecules 
reach the upper regions of the atmosphere, 2) FUV radiation (100-200 nm) break the water molecules (photolysis), 3) XUV radiation $(0.1-100 \mathrm{~nm}$ ) heat up the upper layers of the atmosphere, 4 ) if the thermal velocity exceeds the escape velocity of the hydrogen and oxygen atoms, they can escape the planet. All these steps are considered to occur to compute the mass loss from the planets.

The estimation of the FUV and XUV radiations are very observationally challenging for brown dwarfs. Estimations of the X-ray luminosity exist for M-dwarfs (Pizzolato et al. 2003) and for brown dwarfs (e.g. Williams et al.|2014), but the latter are actually mainly non-detections. Besides, for some cool dwarfs, the Lyman- $\alpha$ emission $(121.6 \mathrm{~nm})$, which is a good proxy for the photolysis wavelength range, can be measured (see Bourrier et al. 2017b for TRAPPIST-1). Very recently for TRAPPIST-1, the closest planet host we have to a brown dwarf, the following values have been obtained with the Space Telescope Imaging Spectrograph (STIS) on HST: $L_{\mathrm{XUV}}=5.26-7.30 \times 10^{26} \mathrm{erg} . \mathrm{s}^{-1}$ and $L_{\alpha}=1.44-1.81 \times 10^{26} \mathrm{erg} . \mathrm{s}^{-1}$ (Bourrier et al. 2017a). The XUV luminosity is approximatively similar to that of Proxima Centauri but the Lyman- $\alpha$ emission is much lower (Bourrier et al. 2017b a). TRAPPIST-1 might be at a transition between active M-dwarfs and more quiet brown dwarfs.

The emission of a M-dwarf and the emission of a brown dwarf have no reason to be similar, and taking into account their difference leads to somewhat different estimations for the water loss. The studies about water loss from planets around lowmass stars and BDs mentioned before differ in the estimations of the high energy radiations. While Barnes and Heller (2013) and Luger and Barnes (2015) considered XUV fluxes measured for early-type M-dwarfs (e.g. Pizzolato et al. 2003), Ribas et al. (2016) and Bolmont et al. (2017) considered more recent estimations of the XUV fluxes for later-type M-dwarfs (Williams et al. 2014, Osten et al. 2015). Another main difference between these studies is that the latter used an estimation of the efficiency $\varepsilon$ of the steps 3 ) and 4) based on 1D radiation-hydrodynamic massloss simulations (Owen and Alvarez 2016).

Fig. 4 shows the results obtained by a) Barnes and Heller (2013) and b) Bolmont et al. (2017). Due to the lack of observations and the uncertainty over the $\varepsilon$ parameter (the efficiency of converting the XUV photons into the kinetic energy of escaping particles), Barnes and Heller (2013) explored the parameter space of the XUV luminosity (via $L_{\mathrm{XUV}} / L_{\mathrm{bol}}$ ) and $\varepsilon$ (see Fig. 4 a). They concluded that the uncertainties are too big to rule in favor or against the presence of water on planets orbiting in the $\mathrm{HZ}$ of brown dwarfs. In contrast, Bolmont et al. (2017) showed that there is a sweet spot for potentially habitable HZ planets: planets which do not lose a lot of water before reaching the $\mathrm{HZ}$ and which spend a long time in the $\mathrm{HZ}$ afterwards. Fig. 4p) shows the parameter space corresponding to this sweet spot. When considering the range of possible XUV fluxes, the sweet spot moves around but is always existing and non negligible for the less massive planets.

To sum up, there is no consensus yet on the question of whether planets around BDs lose their water by the time they reach the $\mathrm{HZ}$ and the next step will likely come from observations. Indeed, now that planets around very low-mass dwarfs are being discovered, the next tests will be to try to constrain their densities or try to detect 
a) Barnes \& Heller 2013

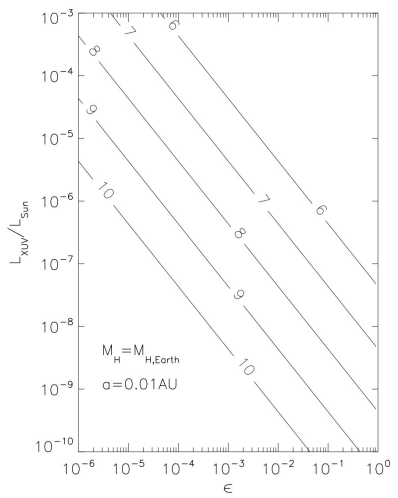

b) Bolmont et al. 2017

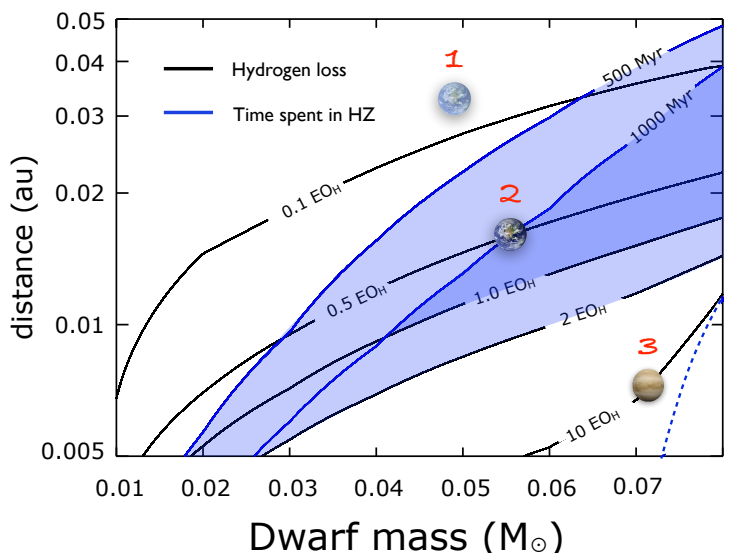

Fig. 4 a) Desiccation timescale for an Earth-like planet orbiting a BD at 0.01 au. Contour lines represent the logarithm of the time for the Earths inventory of hydrogen to be lost (what we call here $\left.1 E O_{H}\right) . \varepsilon$ is the efficiency of converting the XUV photons into the kinetic energy of escaping particles. Figure from Barnes and Heller (2013). b) Hydrogen loss from an Earth-like planet and time spent in the HZ (black and blue contours) for different masses of dwarfs and different planetary orbital distances. The blue shaded areas show the interesting regions of the parameter space: planets in this region lose little hydrogen (little water) before reaching the $\mathrm{HZ}$ and spend a long time in the HZ. Planet 1 loses a very small amount of water but spends less than $500 \mathrm{Myr}$ in the HZ. Planet 2 loses a small amount of water and spends a lot time in the HZ. Planet 3 loses a lot of water and is probably desiccated once it reaches the HZ (unless its initial water reservoir was enormous). Figure adapted from Bolmont et al. (2017)

water in the atmosphere of the very close-in planets. For instance, the masses and densities of the TRAPPIST-1 planets can be estimated with transit timing variations (Gillon et al. 2017, Grimm et al. submitted). A low density gives an indication on the presence of volatiles and the first estimates seem to be pointing in that direction for the TRAPPIST-1 planets. The presence of water on these planets could be an indication that the water loss is overestimated in the studies done so far. In such a context, the future observations of the JWST will be invaluable (Barstow and Irwin 2016; Morley et al. 2017).

\section{In the habitable zone}

Once the hot early phase has passed, one important factor for the eventual appearance of life is the time the planets actually spend inside the HZ. The most important parameters that influence the time a planet spends in the $\mathrm{HZ}$ are the orbital distance of the planet and the mass of the BD: the farther the planet the shorter the time in the HZ (see Figs. 3 and $4 \mathrm{~b}$ ) and the more massive the BD, the longer time in the HZ. 
However the orbital distance of the planet can evolve with time through the tidal interaction between the planet and the $\mathrm{BD}$.

\section{Time spent in the habitable zone vs tidal migration}

Tidal interactions are an important phenomenon that sculpts the architecture of close-in planetary systems. Both the tide raised by the planet in the BD (BD tide) and the tide raised by the BD in the planet (planetary tide) are playing a role in the evolution of the planetary system. Both tides influence the semi-major axis $a$ and eccentricity $e$ of the planet. The planetary tide also influences the planet's rotation period $\Omega_{\mathrm{p}}$ and its obliquity $\varepsilon_{\mathrm{p}}$ (the angle between the planet's rotation axis and the direction of the orbital angular momentum, the obliquity of the Earth is of about $23^{\circ}$ ). The BD tide influences the inclination of the planet (or the BD obliquity $\varepsilon_{\star}$ ) and the rotation $\Omega_{*}$ of the BD. Fig. 5 shows the evolution timescales for the different quantities for a $1 M_{\oplus}$ planet orbiting a $0.04 M_{\odot}$ BD due to the a) planetary tide and b) BD tide.

The evolution timescales depend on the stellar and planetary parameters and the orbit parameters. Among the parameters is the dissipation, which is a measure of how the system loses energy. This parameter is very poorly constrained and depends on the structural and rotational parameters of the body considered (e.g. for stars, Mathis 2015). Most tidal orbital studies use a simple model of equilibrium tide, where the dissipation is parametrized by a single parameter: a time lag $\Delta t$ in the constant time lag model (e.g., Mignard 1979, Hut 1981; Eggleton et al. 1998), or a quality factor $Q$ in the constant phase lag model (e.g., Goldreich and Soter 1966)).

Fig. 3 shows the tidal evolution of planets around a $0.04 M_{\odot}$ BD. These planets are initially on circular orbits, with a zero obliquity and a synchronized rotation so only the BD tide influences their orbital evolution. The planets undergo an important tidal migration, which makes them either fall onto the BD or survive the early evolution and migrate outwards. When the planets are in the HZ, their orbital distance is constant: the evolution timescale due to the BD tide has become so large (due to the small $\mathrm{BD}$ radius) that planets do not significantly migrate over timescales of several gigayears.

Andreeshchev and Scalo (2004) estimated the time a planet orbiting a BD can spend in the habitable zone. However, they did not take into account the tidal interactions between the BD and the planet. Bolmont et al. (2011) and Bolmont (2013) showed that this interaction acts to decrease the time a planet can spend in the HZ. They showed that 1) the higher the BD mass, 2) the higher the dissipation in the BD, 3) the higher the dissipation in the planet, the less time the planet spends in the HZ (See Fig. $4 \mathrm{~b}$ for the effect of the mass of the BD). However, despite that, they show that planets around BDs more massive than $0.04 M_{\odot}$ could stay in the $H Z$ up to a few gigayears (see Figure 6), leaving plenty of time for life to potentially appear and evolve (Bolmont et al. 2011). 
a) Planetary tide timescales

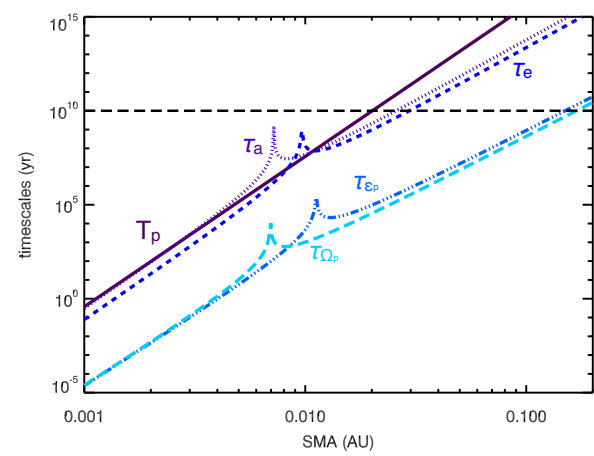

b) Brown dwarf tide timescales

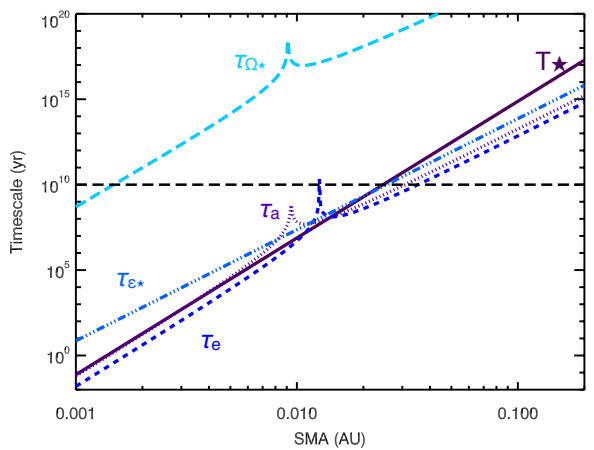

Fig. 5 Evolution timescales of the different quantities $\left(a, e, \Omega_{\mathrm{p}}, \varepsilon_{\mathrm{p}}, \Omega_{*}, \varepsilon_{\star}\right)$ impacted by tides for the a) planetary tide and b) brown dwarf tide. These timescales were calculated for an Earth-mass planet orbiting a 1 Myr old $0.04 M_{\odot}$ BD. The dissipation of the planet is equal to the Earth's (same $\Delta t$, see Neron de Surgy and Laskar 1997) and the dissipation of the BD is taken to be one of a hot Jupiter (Hansen 2010). Figure from Bolmont (2013).

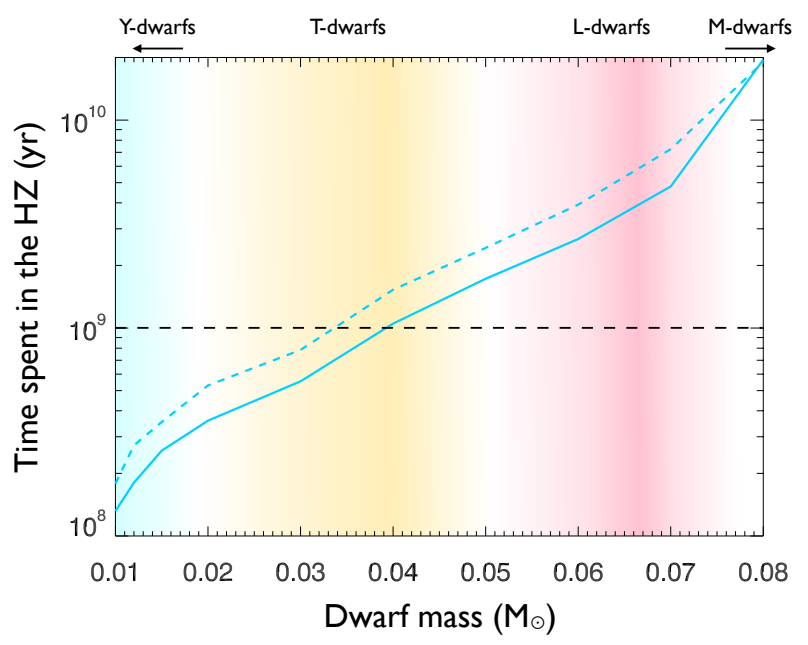

Fig. 6 Maximum time spent in the $\mathrm{HZ}$ for planets orbiting dwarfs of different masses (different spectral type). Figure adapted from Bolmont et al. (2011). 


\section{One planet system vs multiple planet system}

However, once the planets reach the $\mathrm{HZ}$ and assuming they could retain a sufficient part of their initial water reservoir, the presence of surface liquid water is still not yet assured.

Let us first consider that there is only one planet in the system. Fig. 5 shows that by the time close-in planets reach the HZ (after a few $10 \mathrm{Myr}$ to $100 \mathrm{Myr}$, see Fig. 33, planetary tides have had time to damp the initial obliquity, synchronize the rotation and damp the eccentricity so that planets are on a circular orbit, have a zero obliquity and are tidally locked. The planet therefore always shows the same side to the $\mathrm{BD}$ and its poles receive very little light. This raises the problem of the possible existence of regions on the planet where the temperature is constantly lower than the melting point of water and where all the water of the planet will condense (the so called "cold traps", e.g. Joshi 2003). In this configuration, the night side and the poles could be cold traps and the planet would not be able to host surface liquid water.

However, there are mechanisms which can prevent the appearance of cold traps or that can prevent synchronization altogether. For instance, the existence of a dense enough atmosphere would allow a better heat repartition and allow surface liquid water just as Wordsworth et al. (2011) showed for Gliese $581 \mathrm{~d}$ (a super-Earth orbiting a $0.3 M_{\odot}$ dwarf). Also, if planets are synchronized on a close-in orbit, their rotation could trigger winds that would efficiently redistribute heat to the night side (Showman and Polvani 2011, Leconte et al. 2013, Bolmont et al. 2016). Recently, Turbet et al. (2016) and Turbet et al. (2017) respectively showed that Proxima-b and TRAPPIST-1 e, $f, g$ could have surface liquid water despite a synchronous rotation. Furthermore, a high enough geothermal flux could also prevent the appearance of cold traps. Finally, recent works (e.g. Leconte et al. 2015) showed that the atmospheric tides could act to desynchronize the rotation for planets around lowmass stars. Atmospheric tides are different from gravitational tides: the repartition of mass in the atmosphere is due to the irradiation from the star not the gravitational pull of the star (e.g. Gold and Soter 1969). Auclair-Desrotour et al. (2017) showed that the outcome of atmospheric tides actually depends the stability of atmospheric layers close to the ground (only a convective atmosphere can act to desynchronize the rotation). The prediction of the rotation state of $\mathrm{HZ}$ planets is therefore not straightforward. JWST observations could help establish the presence of an atmosphere and distinguish between a convective atmosphere or a stably stratified atmosphere, which would tell us if the planet is likely to be synchronized or not. Note that Leconte et al. (2015) also showed that for stars of mass lower than $0.3 M_{\odot}$, gravitational tides might prevail on atmospheric tides, but this should be investigated further.

The situation differs significantly if the planet is part of a multiple planet system. Due to planet-planet interactions, both eccentricity and obliquity do not tend to 0 but to an equilibrium value which is the result of the competition between planet-planet excitation and tidal damping. 
An extreme case of planet-planet interactions is the Mean Motion Resonance (MMR), which happens when the ratio between the orbital period of two planets is commensurable. One of the consequences is that the eccentricity of both planets is excited to higher levels. A close-by example is the 1:2:4 MMR between Io, Europa and Ganymede. The resonance maintains a non-negligible eccentricity and causes Io to experience an intense internal heating due to the stress it experiences on one orbit. Spencer et al. (2000) estimated the internal heat flux of Io to be around $3 \mathrm{~W} / \mathrm{m}^{2}$. This high heat flux is responsable for the intense volcanic activity (e.g. the Tvashtar volcano, Spencer et al. 2007). To give a point of comparison, the internal heat flux of Earth is about 40 times lower than Io (about $0.08 \mathrm{~W} / \mathrm{m}^{2}$ but mainly due to radioactivity; e.g., Davies and Davies 2010). A tidally evolving planet in the HZ of a BD could thus experience such an intense tidal heating that it can have repercussions on the internal structure of the planet (Mantle overheating, e.g. Běhounková et al.2011 and Henning and Hurford 2014, Effect on the planetary magnetic field, e.g. Driscoll and Barnes 2015) and it can drive the atmosphere of the planet in a runaway greenhouse state. Jackson et al. (2008), Barnes et al. (2009, 2013) investigated this latter phenomenon for planets around M-dwarfs and more massive stars and introduced the notion of "tidal habitable zone" and "tidal Venus" planets: they are in the classical $\mathrm{HZ}$ but have a tidal heat flux higher than $309 \mathrm{~W} \mathrm{~m}^{-2}$, which triggers the runaway greenhouse state.

Bolmont (2013) investigated the effect of tides on the HZ limit around BDs for different eccentricities and different albedos for the planet. Fig. 7 shows the HZ limit for a planet orbiting a $0.04 M_{\odot} \mathrm{BD}$ a) not taking into account tidal heating b) taking into account tidal heating (and assuming a dissipation equal to the Earth's, Neron de Surgy and Laskar 1997). Eccentricity has an effect on the HZ limits: the higher the eccentricity, the farther the HZ limits (see Fig. 7 h). When taking into account tidal heating the $\mathrm{HZ}$ inner limit is strongly impacted. Overall, tidal heating has the effect of narrowing the $\mathrm{HZ}$ by pushing away the inner edger more than the outer edge. While Bolmont (2013) did not investigate the effect of the obliquity, note that a non-zero obliquity would also act as to push and narrow the $\mathrm{HZ}$ even more.

In multiple planet systems, the eccentricity and obliquity can be maintained to high enough levels so that tidal heating has an impact on the potential of the planet to host surface liquid water. Bolmont et al. (2014) extended the works of Barnes et al. (2013) to treat the specific case of multiple planet systems around BD. They illustrated the importance of tides by considering the case of a system of three Earthsized planets orbiting just outside the corotation radius of a $0.08 M_{\star}$ dwarf for two different tidal dissipation factors. The planets experience a convergent outward migration, which leads either to a resonant capture for a high BD dissipation or not for low dissipation. In the case of a high BD dissipation, they found that the planets enter a MMR chain (1:2:4) in a few million years of evolution.

Fig. 8 shows the short term evolution of such a system at an age of $1 \mathrm{Gyr}$ for the two cases. The two inner planets are in the HZ. The eccentricities of the planets in case a) are relatively small $<0.07$ but in case b) due to the MMR excitation, they can reach 0.15 . In case a), the average of the tidal heat flux of the inner planet remains below the runaway greenhouse limit. In case b), its tidal heat flux is almost 
a) No tidal heating in planet

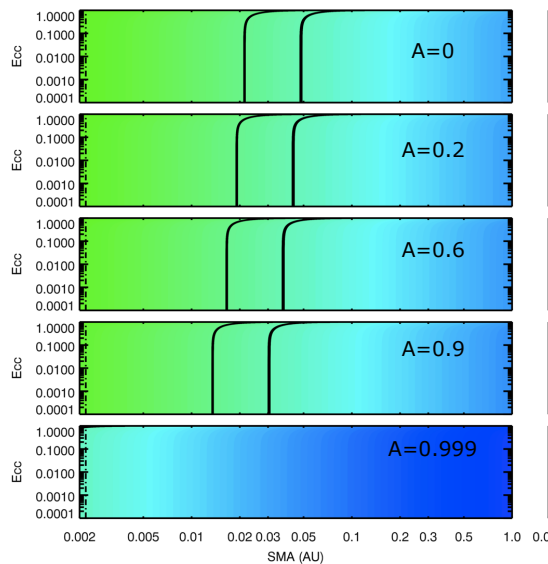

b) Tidal heating in planet

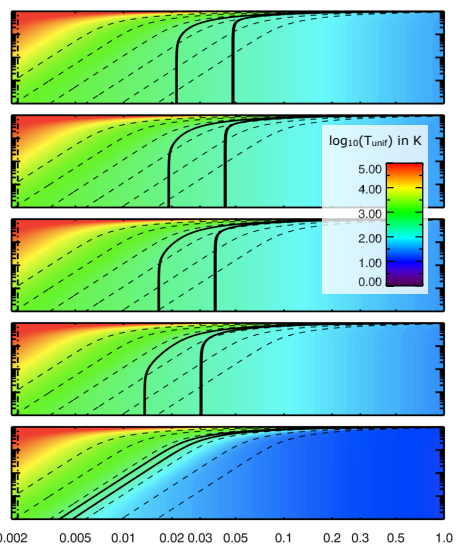

Fig. 7 Maps of the $\log _{10}$ of the temperature of a $1 M_{\oplus}$ planet orbiting a 100 Myr old $0.04 M_{\odot}$ $\mathrm{BD}$ as a function of its orbital distance and the eccentricity of its orbit. a) The tidal heating is not taken into account. b) The tidal heating is taken into account. The different panels are for a different albedo of the planet (A increases from top to bottom). The two black lines correspond to temperatures of $180 \mathrm{~K}$ (flux of $240 \mathrm{~W} \cdot \mathrm{m}^{-2}$ ) and $270 \mathrm{~K}$ (flux of $300 \mathrm{~W} \cdot \mathrm{m}^{-2}$ ), which crudely represent the limits of the HZ. Figure adapted from Bolmont (2013).
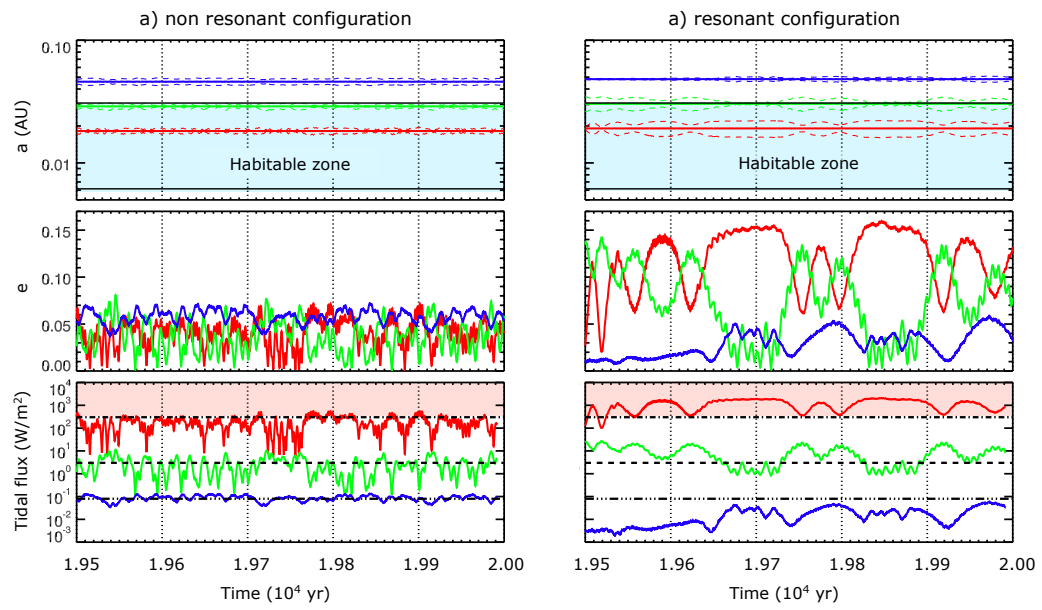

Fig. 8 Evolution of the orbital distance, eccentricity and tidal heat flux of three Earth-sized planets orbiting a BD of $0.08 M_{\star}$ in a) a non-resonant configuration, b) a resonant configuration (1:2 MMR). Top graph: the full colored lines correspond to the semi-major axis evolution of the 3 planets, and the dashed lines correspond to their perihelion and aphelion distances. The blue shaded region is the HZ. Middle graph: eccentricity of the 3 planets. Bottom graph: the full colored lines correspond to the tidal heat flux of the 3 planets. The black dashed dotted line corresponds to the limit of runaway greenhouse (e.g. Kopparapu 2013), the dashed line corresponds to Io's heat flux and the dashed 3 dots line corresponds to Earth's heat flux. The shaded red region corresponds to where the heat flux is so high that the planet is in a runaway greenhouse state. Figure from Bolmont et al. (2014). 
always above the runaway greenhouse limit: in spite of being in the HZ, it would therefore be a "tidal Venus" and would be too hot to host surface liquid water. The middle planet (in green), also in the HZ, with a flux higher than Io's in case b) would experience a intense volcanism, which could be problematic for potential life. Conversely, this planet spends some time around aphelion outside the insolation HZ and could be too cold to be able to sustain a potential liquid water reservoir. However taking into account tidal heating could improve the conditions for habitability at apocenter. One could imagine a more extreme case of a planet on an orbit completely outside the HZ but heated up by tides sufficiently to be able to host surface liquid water.

This mechanism can facilitate the habitable conditions for planets on the outer edge of the HZ or even exterior to the HZ. Recently, Ramirez \& Kaltenegger 2017 showed that volcanoes ejecting hydrogen in the atmosphere in a regular way could contribute to extend the HZ farther than the classical limits. Such volcanism maintained by tides in a multi-planet system could therefore be favorable to surface liquid water conditions in the colder regions of a system.

Therefore, when assessing habitability of planets in the HZ of BDs, one should investigate if tides are strong enough to drive a runaway greenhouse (Barnes et al. 2013: Bolmont et al. 2014). If the planet absorbs an average flux $\left(\Phi_{\star}+\Phi_{\text {tides }}\right)$ lower than the greenhouse limit, the planet can sustain a liquid water reservoir, but if it receives an average flux higher than the greenhouse limit the planet will be too hot to be able to sustain a liquid water reservoir.

\section{Brown dwarfs' variability}

Some brown dwarfs are known to be variable objects at various wavelengths: from near-IR (e.g. Artigau et al. 2009, thought to be due to clouds or spots) to X-ray (Rutledge et al. 2000). While the photometric variability is of relatively low amplitude (Buenzli et al. 2014) and probably does not have a significant impact on the atmosphere, the energetic flares can potentially have a negative effect on the atmosphere (driving water loss as during the runaway greenhouse phase, see previous section) and life (e.g. Tabataba-Vakili et al.|2016 for M-dwarfs).

The effect of energetic flares has been widely discussed for M-dwarfs. For instance, UV flares can lead to ozone depletion, which increases the penetration of the UV photons and can damage eventual surface life (Segura et al.2010). Depending on their frequency, the flares can also alter the chemistry of the planet preventing it from reaching an equilibrium (Venot et al. 2016, Segura et al. 2010). Recently, Vida et al. (2017) and O'Malley-James and Kaltenegger (2017) estimated that the UV environment of the TRAPPIST-1 system might be too harsh for life.

However, brown dwarfs might be quieter than M-dwarfs (Mohanty et al. 2002, Williams et al. 2014). Besides, the measured activity in itself might not be representative of what the planet actually receives during flaring events: the planet is impacted only if the flare is in its direction (Segura et al.2010). Furthermore, the effect 
of these energetic radiations also strongly depends on the atmosphere of the planet (e.g. O'Malley-James and Kaltenegger 2017) and its magnetic field (e.g. Kay et al. 2016). Finally, despite all the drawbacks of receiving energetic radiations, Ranjan et al. (2017) also pointed out that some UV might be required for the origin of life.

The questions of the influence of harmful flares on the planetary environment of planets around cool dwarfs will benefit both from the future observations of the atmospheric chemistry of these objects and the necessary constraints on the dwarf's complete spectrum.

\section{Observational perspectives for planets around brown dwarfs (and more generally cool dwarfs)}

The future prospects of observation and characterization of planets around BD have never been better (He et al. 2017). A few missions are either dedicated to planets orbiting very faint objects: TRAPPIST (Gillon et al. 2011), SPECULOOS (Gillon et al.2013) and SPIRou (Artigau et al.2011); or able to observe them such as the K2 mission (Haas et al. 2014) and Spitzer (as proposed by Triaud et al.|2013, based on a study of Belu et al. 2013).

The recent discovery of the multiple planet system around TRAPPIST-1 (Gillon et al. 2016, 2017) highlights the importance of studying these objects. TRAPPIST-1 is a quasi BD, just above the theoretical limit between BDs and M-dwarfs, and illustrates the fact that planets will probably be found around BDs in the near future (e.g., with SPECULOOS, Gillon et al. 2013). Planetary systems around very low mass stars and BDs (hereafter ultra-cool dwarfs) are dynamically rich: the planets are tidally evolving, most systems are compact and therefore planet-planet interactions play a major role.

What makes the planetary systems orbiting ultra-cool dwarfs even more interesting is the prospect of future observations. Indeed the planets in the HZ of cool dwarfs are the only $\mathrm{HZ}$ planets whose atmosphere can be probed by telescopes such as the JWST (Belu et al. 2013). For instance, Barstow and Irwin (2016) recently showed that ozone could be detected in the atmosphere of the three inner planets of TRAPPIST-1 with a high number of transits (at least 60 for TRAPPIST-1c). Morley et al. (2017) also showed that it could be potentially possible to differentiate between an Earth-like, a Venus-like and a Titan-like atmosphere with JWST observations of TRAPPIST-1c. For non-transiting planets, there are also possibilities via emission spectroscopy with the E-ELT and emission phase curves with the JWST (see Turbet et al.|2016, for a discussion about Proxima-b).

The era of planets around BDs is almost upon us and these objects will represent a highly interesting scientific domain. Rocky planets around BDs (and very low-mass stars, such as the TRAPPIST-1 planetary system), will allow us to do comparative planetology and explore in a unique way the effect of tidal orbital dynamics on the potential climate of these planets. 
Acknowledgements E.B. acknowledges funding by the European Research Council through ERC grant SPIRE 647383. This research has made use of NASA's Astrophysics Data System.

\section{References}

Allard F (2014) The BT-Settl Model Atmospheres for Stars, Brown Dwarfs and Planets. In: Booth M, Matthews BC Graham JR (eds) Exploring the Formation and Evolution of Planetary Systems, IAU Symposium, vol 299, pp 271-272, DOI 10.1017/S1743921313008545

Andreeshchev A Scalo J (2004) Habitability of Brown Dwarf Planets. In: R Norris \& F Stootman (ed) Bioastronomy 2002: Life Among the Stars, IAU Symposium, vol 213, pp 115-+

Anglada-Escudé G, Amado PJ, Barnes J et al. (2016) A terrestrial planet candidate in a temperate orbit around Proxima Centauri. Nature536:437-440

Artigau É, Bouchard S, Doyon R Lafrenière D (2009) Photometric Variability of the T2.5 Brown Dwarf SIMP J013656.5+093347: Evidence for Evolving Weather Patterns. ApJ701:1534-1539

Artigau É, Donati JF Delfosse X (2011) Planet Detection, Magnetic Field of Protostars and Brown Dwarfs Meteorology with SPIRou. In: Johns-Krull C, Browning MK West AA (eds) 16th Cambridge Workshop on Cool Stars, Stellar Systems, and the Sun, Astronomical Society of the Pacific Conference Series, vol 448, p 771

Auclair-Desrotour P, Laskar J Mathis S (2017) Atmospheric tides in Earth-like planets. A\&A603:A107

Barnes R Heller R (2013) Habitable Planets Around White and Brown Dwarfs: The Perils of a Cooling Primary. Astrobiology 13:279-291

Barnes R, Raymond SN, Jackson B Greenberg R (2008) Tides and the Evolution of Planetary Habitability. Astrobiology 8:557-568

Barnes R, Jackson B, Greenberg R Raymond SN (2009) Tidal Limits to Planetary Habitability. ApJ700:L30-L33

Barnes R, Jackson B, Greenberg R, Raymond SN Heller R (2010) Tidal Constraints on Planetary Habitability. In: Coudé du Foresto V, Gelino DM Ribas I (eds) Pathways Towards Habitable Planets, Astronomical Society of the Pacific Conference Series, vol 430, p 133

Barnes R, Meadows VS, Domagal-Goldman SD et al. (2011) Habitability of Planets Orbiting Cool Stars. In: Johns-Krull C, Browning MK West AA (eds) 16th Cambridge Workshop on Cool Stars, Stellar Systems, and the Sun, Astronomical Society of the Pacific Conference Series, vol 448, p 391

Barnes R, Mullins K, Goldblatt C et al. (2013) Tidal Venuses: Triggering a Climate Catastrophe via Tidal Heating. Astrobiology 13:225-250

Barrado y Navascués D, Stauffer JR, Briceño C et al. (2001) Very Low-Mass Stars and Brown Dwarfs of the Young Open Cluster IC 2391. ApJS 134:103-114

Barstow JK Irwin PGJ (2016) Habitable worlds with JWST: transit spectroscopy of the TRAPPIST1 system? MNRAS461:L92-L96

Belu AR, Selsis F, Raymond SN et al. (2013) Habitable Planets Eclipsing Brown Dwarfs: Strategies for Detection and Characterization. ApJ 768:125

Bolmont E (2013) Evolution et habitabilité de systèmes planétaires autour d'étoiles de faible masse et de naines brunes. PhD thesis, Université de Bordeaux 1, Université de Bordeaux 1, Pessac, an optional note

Bolmont E, Raymond SN Leconte J (2011) Tidal evolution of planets around brown dwarfs. A \& A 535:A94

Bolmont E, Raymond SN Selsis F (2014) Dynamics of exoplanetary systems, links to their habitability. In: Ballet J, Martins F, Bournaud F, Monier R Reylé C (eds) SF2A-2014: Proceedings of the Annual meeting of the French Society of Astronomy and Astrophysics, pp 63-68

Bolmont E, Libert AS, Leconte J Selsis F (2016) Habitability of planets on eccentric orbits: Limits of the mean flux approximation. A\&A591:A106 
Bolmont E, Selsis F, Owen JE et al. (2017) Water loss from terrestrial planets orbiting ultracool dwarfs: implications for the planets of TRAPPIST-1. MNRAS464:3728-3741

Bonfils X, Delfosse X, Udry S et al. (2013) The HARPS search for southern extra-solar planets. XXXI. The M-dwarf sample. A\&A 549:A109

Boss AP, Butler RP, Hubbard WB et al. (2007) Working Group on Extrasolar Planets. Transactions of the International Astronomical Union, Series A 26:183-186

Bourrier V, de Wit J, Bolmont E et al. (2017a) Temporal Evolution of the High-energy Irradiation and Water Content of TRAPPIST-1 Exoplanets. AJ154:121

Bourrier V, Ehrenreich D, Wheatley PJ et al. (2017b) Reconnaissance of the TRAPPIST-1 exoplanet system in the Lyman- $\alpha$ line. A\&A599:L3

Buenzli E, Apai D, Radigan J, Reid IN Flateau D (2014) Brown Dwarf Photospheres are Patchy: A Hubble Space Telescope Near-infrared Spectroscopic Survey Finds Frequent Low-level Variability. ApJ782:77

Burrows A, Marley MS Sharp CM (2000) The Near-Infrared and Optical Spectra of Methane Dwarfs and Brown Dwarfs. ApJ531:438-446

Běhounková M, Tobie G, Choblet G Čadek O (2011) Tidally Induced Thermal Runaways on Extrasolar Earths: Impact on Habitability. ApJ 728:89

Caballero JA, Béjar VJS, Rebolo R et al. (2007) The substellar mass function in $\sigma$ Orionis. II. Optical, near-infrared and IRAC/Spitzer photometry of young cluster brown dwarfs and planetarymass objects. A \& A 470:903-918

Chabrier G (2002) The Galactic Disk Mass Budget. II. Brown Dwarf Mass Function and Density. ApJ567:304-313

Chabrier G Baraffe I (1997) Structure and evolution of low-mass stars. A \& A 327:1039-1053

Chabrier G Baraffe I (2000) Theory of Low-Mass Stars and Substellar Objects. ARA\&A 38:337377

Comerón F, Neuhäuser R Kaas AA (2000) Probing the brown dwarf population of the Chamaeleon I star forming region. A\&A 359:269-288

Cushing MC, Kirkpatrick JD, Gelino CR et al. (2011) The Discovery of Y Dwarfs using Data from the Wide-field Infrared Survey Explorer (WISE). ApJ 743:50

Davies JH Davies DR (2010) Earth's surface heat flux. Solid Earth 1(1):5-24, URL http:// www.solid-earth.net/1/5/2010/

Desidera S (1999) Properties of Hypothetical Planetary Systems around the Brown Dwarf Gliese 229B. PASP111:1529-1538

Dittmann JA, Irwin JM, Charbonneau D et al. (2017) A temperate rocky super-Earth transiting a nearby cool star. Nature544:333-336

Dressing CD Charbonneau D (2013) The Occurrence Rate of Small Planets around Small Stars. ApJ 767:95

Dressing CD Charbonneau D (2015) The Occurrence of Potentially Habitable Planets Orbiting M Dwarfs Estimated from the Full Kepler Dataset and an Empirical Measurement of the Detection Sensitivity. ApJ807:45

Driscoll PE Barnes R (2015) Tidal Heating of Earth-like Exoplanets around M Stars: Thermal, Magnetic, and Orbital Evolutions. Astrobiology 15:739-760

Eggleton PP, Kiseleva LG Hut P (1998) The Equilibrium Tide Model for Tidal Friction. ApJ 499:853-+

Gillon M, Jehin E, Magain P et al. (2011) TRAPPIST: a robotic telescope dedicated to the study of planetary systems. In: European Physical Journal Web of Conferences, European Physical Journal Web of Conferences, vol 11, p 06002, DOI 10.1051/epjconf/20101106002

Gillon M, Jehin E, Delrez L et al. (2013) SPECULOOS: Search for habitable Planets EClipsing ULtra-cOOl Stars. In: Protostars and Planets VI Posters

Gillon M, Jehin E, Lederer SM et al. (2016) Temperate Earth-sized planets transiting a nearby ultracool dwarf star. Nature 533:221-224

Gillon M, Triaud A, Demory BO et al. (2017) Seven temperate terrestrial planets around the nearby ultracool dwarf star TRAPPIST-1. Nature542:456-460

Gold T Soter S (1969) Atmospheric Tides and the Resonant Rotation of Venus. Icarus11:356-366 
Goldreich P Soter S (1966) Q in the Solar System. Icarus 5:375-389

Haas MR, Barclay T, Batalha NM et al. (2014) The Kepler Mission on Two Reaction Wheels is K2. In: American Astronomical Society Meeting Abstracts \#223, American Astronomical Society Meeting Abstracts, vol 223, p 228.01

Hansen BMS (2010) Calibration of Equilibrium Tide Theory for Extrasolar Planet Systems. ApJ 723:285-299

Hayashi C Nakano T (1963) Evolution of Stars of Small Masses in the Pre-Main-Sequence Stages. Progress of Theoretical Physics 30:460-474

He MY, Triaud AHMJ Gillon M (2017) First limits on the occurrence rate of short-period planets orbiting brown dwarfs. MNRAS464:2687-2697

Hennebelle P Chabrier G (2008) Analytical Theory for the Initial Mass Function: CO Clumps and Prestellar Cores. ApJ 684:395-410

Henning WG Hurford T (2014) Tidal Heating in Multilayered Terrestrial Exoplanets. ApJ789:30

Henry TJ, Jao WC, Winters JG et al. (2016) The Census of Objects within 10 Parsecs. In: American Astronomical Society Meeting Abstracts, American Astronomical Society Meeting Abstracts, vol 227, p 142.01

Hut P (1981) Tidal evolution in close binary systems. A \& A 99:126-140

Jackson B, Barnes R Greenberg R (2008) Tidal heating of terrestrial extrasolar planets and implications for their habitability. MNRAS391:237-245

Joshi M (2003) Climate Model Studies of Synchronously Rotating Planets. Astrobiology 3:415427

Joshi MM Haberle RM (2012) Suppression of the Water Ice and Snow Albedo Feedback on Planets Orbiting Red Dwarf Stars and the Subsequent Widening of the Habitable Zone. Astrobiology $12: 3-8$

Kasting JF, Whitmire DP Reynolds RT (1993) Habitable Zones around Main Sequence Stars. Icarus 101:108-128

Kay C, Opher M Kornbleuth M (2016) Probability of CME Impact on Exoplanets Orbiting M Dwarfs and Solar-like Stars. ApJ826:195

Kirkpatrick JD (2013) Cold brown dwarfs with WISE: Y dwarfs and the field mass function. Astronomische Nachrichten 334:26-31

Kirkpatrick JD, Reid IN, Liebert J et al. (1999) Dwarfs Cooler than "M": The Definition of Spectral Type "L" Using Discoveries from the 2 Micron All-Sky Survey (2MASS). ApJ 519:802-833

Kopparapu RK (2013) A Revised Estimate of the Occurrence Rate of Terrestrial Planets in the Habitable Zones around Kepler M-dwarfs. ApJL 767:L8

Kumar SS (1963) The Structure of Stars of Very Low Mass. ApJ 137:1121

Lammer H, Selsis F, Ribas I et al. (2003) Atmospheric Loss of Exoplanets Resulting from Stellar X-Ray and Extreme-Ultraviolet Heating. ApJ1 598:L121-L124

Leconte J, Baraffe I, Chabrier G, Barman T Levrard B (2009) Structure and evolution of the first CoRoT exoplanets: probing the brown dwarf/planet overlapping mass regime. A \& A 506:385389

Leconte J, Chabrier G, Baraffe I Levrard B (2011a) The radius anomaly in the planet/brown dwarf overlapping mass regime. Detection and Dynamics of Transiting Exoplanets, St Michel l'Observatoire, France, Edited by F Bouchy; R Diaz; C Moutou; EPJ Web of Conferences, Volume 11, id03004 11:3004-+

Leconte J, Lai D Chabrier G (2011b) Distorted, nonspherical transiting planets: impact on the transit depth and on the radius determination. A \& A 528:A41+

Leconte J, Forget F, Charnay B et al. (2013) 3D climate modeling of close-in land planets: Circulation patterns, climate moist bistability, and habitability. A\&A554:A69

Leconte J, Wu H, Menou K Murray N (2015) Asynchronous rotation of Earth-mass planets in the habitable zone of lower-mass stars. Science 347:632-635

López Martí B, Eislöffel J, Scholz A Mundt R (2004) The brown dwarf population in the Chamaeleon I cloud. A\&A 416:555-576

Luger R Barnes R (2015) Extreme Water Loss and Abiotic O2Buildup on Planets Throughout the Habitable Zones of M Dwarfs. Astrobiology 15:119-143 
Luhman KL (2012) The Formation and Early Evolution of Low-Mass Stars and Brown Dwarfs. ARA\&A50:65-106

Mathis S (2015) Variation of tidal dissipation in the convective envelope of low-mass stars along their evolution. A\&A580:L3

Mignard F (1979) The evolution of the lunar orbit revisited. I. Moon and Planets 20:301-315

Mohanty S, Basri G, Shu F, Allard F Chabrier G (2002) Activity in Very Cool Stars: Magnetic Dissipation in Late M and L Dwarf Atmospheres. ApJ 571:469-486

Morata O, Palau A, González RF et al. (2015) First Detection of Thermal Radiojets in a Sample of Proto-brown Dwarf Candidates. ApJ807:55

Morley CV, Kreidberg L, Rustamkulov Z, Robinson T Fortney JJ (2017) Observing the Atmospheres of Known Temperate Earth-sized Planets with JWST. ArXiv e-prints

Muirhead PS, Johnson JA, Apps K et al. (2012) Characterizing the Cool KOIs. III. KOI 961: A Small Star with Large Proper Motion and Three Small Planets. ApJ 747:144

Nakajima T, Oppenheimer BR, Kulkarni SR et al. (1995) Discovery of a cool brown dwarf. Nature 378:463-465

Neron de Surgy O Laskar J (1997) On the long term evolution of the spin of the Earth. A \& A 318:975-989

O'Malley-James JT Kaltenegger L (2017) UV surface habitability of the TRAPPIST-1 system. MNRAS469:L26-L30

Osten RA, Melis C, Stelzer B et al. (2015) The Deepest Constraints on Radio and X-Ray Magnetic Activity in Ultracool Dwarfs from WISE J104915.57-531906.1. ApJ805:L3

Owen JE Alvarez MA (2016) UV Driven Evaporation of Close-in Planets: Energy-limited, Recombination-limited, and Photon-limited Flows. ApJ816:34

Padoan P Nordlund ^̊ (2004) The "Mysterious" Origin of Brown Dwarfs. ApJ 617:559-564

Phan-Bao N, Guibert J, Crifo F et al. (2001) New neighbours: IV. 30 DENIS late-M dwarfs between 15 and 30 parsecs. A\&A 380:590-598

Pizzolato N, Maggio A, Micela G, Sciortino S Ventura P (2003) The stellar activity-rotation relationship revisited: Dependence of saturated and non-saturated X-ray emission regimes on stellar mass for late-type dwarfs. A\&A 397:147-157

Ranjan S, Wordsworth R Sasselov DD (2017) The Surface UV Environment on Planets Orbiting M Dwarfs: Implications for Prebiotic Chemistry and the Need for Experimental Follow-up. ApJ843:110

Rauer H, Gebauer S, Paris PV et al. (2011) Potential biosignatures in super-Earth atmospheres. I. Spectral appearance of super-Earths around M dwarfs. A\&A529:A8

Rebolo R, Zapatero Osorio MR Martín EL (1995) Discovery of a brown dwarf in the Pleiades star cluster. Nature 377:129-131

Ribas I, Bolmont E, Selsis F et al. (2016) The habitability of Proxima Centauri b. I. Irradiation, rotation and volatile inventory from formation to the present. A\&A596:A111

Rodler F López-Morales M (2014) Feasibility Studies for the Detection of $\mathrm{O}_{2}$ in an Earth-like Exoplanet. ApJ781:54

Rutledge RE, Basri G, Martín EL Bildsten L (2000) Chandra Detection of an X-Ray Flare from the Brown Dwarf LP 944-20. ApJ538:L141-L144

Salpeter EE (1955) The Luminosity Function and Stellar Evolution. ApJ121:161

Segura A, Kasting JF, Meadows V et al. (2005) Biosignatures from Earth-Like Planets Around M Dwarfs. Astrobiology 5:706-725

Segura A, Walkowicz LM, Meadows V, Kasting J Hawley S (2010) The Effect of a Strong Stellar Flare on the Atmospheric Chemistry of an Earth-like Planet Orbiting an M Dwarf. Astrobiology 10:751-771

Selsis F, Kasting JF, Levrard B et al. (2007) Habitable planets around the star Gliese 581? A \& A 476:1373-1387

Showman AP Polvani LM (2011) Equatorial Superrotation on Tidally Locked Exoplanets. ApJ738:71

Skrutskie MF, Cutri RM, Stiening R et al. (2006) The Two Micron All Sky Survey (2MASS). AJ 131:1163-1183 
Spencer JR, Jessup KL, McGrath MA, Ballester GE Yelle R (2000) Discovery of Gaseous $\mathrm{S}_{2}$ in Io's Pele Plume. Science 288:1208-1210

Spencer JR, Stern SA, Cheng AF et al. (2007) Io Volcanism Seen by New Horizons: A Major Eruption of the Tvashtar Volcano. Science 318:240

Spiegel DS, Burrows A Milsom JA (2011) The Deuterium-burning Mass Limit for Brown Dwarfs and Giant Planets. ApJ 727:57-+

Tabataba-Vakili F, Grenfell JL, Grießmeier JM Rauer H (2016) Atmospheric effects of stellar cosmic rays on Earth-like exoplanets orbiting M-dwarfs. A\&A585:A96

Triaud AHMJ, Gillon M, Selsis F et al. (2013) A search for rocky planets transiting brown dwarfs. ArXiv e-prints

Turbet M, Leconte J, Selsis F et al. (2016) The habitability of Proxima Centauri b. II. Possible climates and observability. A\&A596:A112

Turbet M, Bolmont E, Leconte $\mathrm{J}$ et al. (2017) Climate diversity on cool planets around cool stars with a versatile 3-D Global Climate Model: the case of TRAPPIST-1 planets. ArXiv e-prints

Venot O, Rocchetto M, Carl S, Roshni Hashim A Decin L (2016) Influence of Stellar Flares on the Chemical Composition of Exoplanets and Spectra. ApJ830:77

Vida K, Kővári Z, Pál A, Oláh K Kriskovics L (2017) Frequent Flaring in the TRAPPIST-1 System - Unsuited for Life? ApJ841:124

Watson AJ, Donahue TM Walker JCG (1981) The dynamics of a rapidly escaping atmosphere Applications to the evolution of earth and Venus. Icarus48:150-166

Williams PKG, Cook BA Berger E (2014) Trends in Ultracool Dwarf Magnetism. I. X-Ray Suppression and Radio Enhancement. ApJ785:9

Wordsworth RD, Forget F, Selsis F et al. (2011) Gliese 581d is the First Discovered Terrestrialmass Exoplanet in the Habitable Zone. ApJ Lett 733:L48+

Zapatero Osorio MR, Rebolo R, Martin EL et al. (1997) New Brown Dwarfs in the Pleiades Cluster. ApJ Lett 491:L81 